\title{
Patient-Specific Risk Factors Exist for Hip Fractures After Arthroscopic Femoroacetabular Impingement Surgery, But Not for Dislocation-An Analysis of More Than 25,000 Hip Arthroscopies
}

\author{
Kunal Varshneya, B.S., Geoffrey D. Abrams, M.D., Seth L. Sherman, M.D., and \\ Marc R. Safran, M.D.
}

Purpose: To identify postoperative complications and risk factors associated with hip fracture and dislocation following primary arthroscopic surgical management of femoroacetabular impingement (FAI) syndrome. Methods: MarketScan was queried to identify patients who underwent FAI surgery from 2007 to 2016. Patients were stratified into 2 groups: acetabuloplasty only or femoroplasty only. A subanalysis of combined acetabuloplasty and femoroplasty also was undertaken. Surgical outcomes were followed postoperatively for 180 days. Multivariate logistic regression was used to control for with an alpha value of 0.05 set as significant. Results: This study identified 13,809 patients (mean age, 36.3 years) who underwent primary acetabuloplasty or femoroplasty. We also identified 10,026 patients who underwent both procedures. Postoperative complication rates were similar between the cohorts (acetabuloplasty 17.1\%, femoroplasty $19.9 \%, P=.0622$ ). Rates of hip fracture (femoroplasty: $2.4 \%$ vs acetabuloplasty: $2.0 \%, P=.0302$ ) and heterotopic ossification (femoroplasty: $11.3 \%$ vs acetabuloplasty: $8.8 \%, P<.0001$ ) were greater in the femoroplasty-only cohort. Combined acetabuloplasty and femoroplasty was associated with the greatest complication burden of $21.6 \%(P<.0001)$. After multivariate regression, differences in age, sex, comorbid status, or procedure type did not influence odds in risk for postoperative hip dislocation. Adjusted data showed that neither femoroplasty nor acetabuloplasty influenced odds of hip fracture $(P>.05)$. Patients who were aged younger than 20 years old were significantly less likely to fracture their hips postoperatively than patients aged $60+$ years (odds ratio $0.3,95 \%$ confidence interval 0.1-0.8). Hypertension was independently associated with increased odds of hip fracture (odds ratio 1.7, 95\% confidence interval 1.2-3.5). Conclusions: Older age, male sex, and hypertension all carry increased risk for a hip fracture following acetabuloplasty or femoroplasty. Patient- and procedure-specific factors that could be assessed with this database did not influence risk for hip dislocation. Level of Evidence: Level III; retrospective comparative observation trial.

\footnotetext{
From the Department of Orthopaedic Surgery, Stanford University Medical Center, Palo Alto, California, U.S.A.

The authors report the following potential conflicts of interest or sources of funding: M.R.S reports American Journal of Sports Medicine: editorial or governing board; Biomimedica: stock or stock options; unpaid consultant; DJ Orthopaedics: IP royalties; International Society for Hip Arthroscopy and International Society of Arthroscopy, Knee Surgery, and Orthopaedic Sports Medicine: board or committee member; JISAKOS and Journal of Hip Preservation Surgery: editorial or governing board; Medacta: paid consultant; paid presenter or speaker; Saunders/Mosby-Elsevier: publishing royalties, financial or material support; Smith $\theta$ Nephew: IP royalties; paid presenter or speaker, research support; Stryker: IP royalties; and Wolters Kluwer HealthLippincott Williams $\theta$ Wilkins: publishing royalties, financial or material support. S.L.S. reports ACL Study Group: board or committee member; American Journal of Orthopedics: editorial or governing board; American Orthopaedic Society for Sports Medicine: board or committee member; Arthrex: paid consultant, research support; Arthroscopy: editorial or governing board; Arthroscopy Association of North America: board or committee member; Ceterix Orthopaedics: paid consultant; CONMED Linvatec: paid consultant; Flexion Therapeutics: paid consultant; GLG Consulting: paid consultant; International Cartilage Regeneration $\theta$ Joint Preservation Society: board or
}

committee member; International Society of Arthroscopy, Knee Surgery, and Orthopaedic Sports Medicine: board or committee member; JRF Ortho: paid consultant; Moximed: paid consultant; Olympus: paid consultant; and Vericel: paid consultant. G.D.A. reports AAOS: board or committee member; American Orthopaedic Society for Sports Medicine: board or committee member; Arthrex: other financial or material support; Cytonics: paid consultant, stock or stock options; Fidia Pharma: paid consultant; International Society of Arthroscopy, Knee Surgery, and Orthopaedic Sports Medicine: board or committee member; RubiconMD: paid consultant; Sideline Sports Doc: paid consultant; Stryker: other financial or material support; and TeachAIDS: unpaid consultant. Full ICMJE author disclosure forms are available for this article online, as supplementary material.

Received February 15, 2021; accepted November 13, 2021.

Address correspondence to Marc R. Safran, M.D., Department of Orthopaedic Surgery, Stanford University, 450 Broadway St., M/C 6120, Redwood City,CA 94063.E-mail:msafran@stanford.edu

(C) 2021 Published by Elsevier Inc. on behalf of the Arthroscopy Association of North America. This is an open access article under the CC BY-NC-ND license (http://creativecommons.org/licenses/by-nc-nd/4.0/).

2666-061X/21108

https://doi.org/10.1016/j.asmr.2021.11.011 
$\mathbf{F}$ emoral osteoplasty and acetabuloplasty have become prevalent arthroscopic hip procedures in recent years. ${ }^{1-3}$ These procedures are primarily performed to treat symptomatic femoroacetabular impingement (FAI), with femoral osteoplasty being used in cases of cam impingement and acetabuloplasty in cases of pincer impingement. These procedures also are used often in conjunction to treat the most common type of FAI, mixed-type impingement. ${ }^{4-9}$ Given the increasing use of femoral osteoplasty and acetabuloplasty, understanding the risks, and particularly risk factors, for complications of these procedures is paramount. Complications of hip arthroscopy have been well documented, and the procedures are commonly understood to carry low complication rates, with overall complication rates between $1 \%$ and $8 \% .^{10-12}$ Major complications of arthroscopic FAI treatment include proximal femoral fracture, hip dislocation, and avascular necrosis of the femoral head. ${ }^{12-17}$ The documented risk factors for proximal femoral fracture following FAI surgery include over-resection of the femoral head-neck junction, ${ }^{18-21}$ early/excessive postoperative weightbearing, ${ }^{16,22-25}$ and trauma, such as falling on the surgical hip. ${ }^{26-28}$ Risk factors for hip dislocation include excessive acetabular resection, ${ }^{11,29,30}$ ligamentous laxity, ${ }^{31,32}$ iliopsoas tenotomy, ${ }^{33}$ and excessive capsular resection. ${ }^{34-36}$ Many of the identified risk factors are related to operative approach or technique and postoperative management, but the influence of patient specific characteristics on the risk of complications after hip arthroscopy is not well understood.

Using a national claims database, the purpose of this study was to identify postoperative complications and risk factors associated with hip fracture and dislocation following primary arthroscopic surgical management of FAI syndrome. The hypothesis is that surgical technique, comorbidity burden, and increasing age would influence the rates of postoperative hip dislocation and fracture.

\section{Methods}

\section{Data Source}

This study obtained a sample of the MarketScan Commercial Claims and Encounters database (Truven Health Analytics, Ann Arbor, MI) from January 2007 to December 2016. This database is a collection of commercial inpatient, outpatient, and pharmaceutical claims of more than 75 million employees, retirees, and dependents representing a substantial portion of the U.S. population covered by employer-sponsored insurance. MarketScan contains 53 million patient inpatient records, 40 million with employer-sponsored insurance, 3.7 million with Medicare Part B, and 6.8 million on Medicaid, for a total of more than 28 billion patient records. The data are updated quarterly, with all new records becoming available within 15 months of service and $91 \%$ of claims available within 5 months. Due to MarketScan's sourcing from large employers, its data boasts superior longitudinal tracking of patients. Compared with other more frequently used databases in the orthopaedic literature such as PearlDiver (PearlDiver Technologies Inc., Colorado Springs, CO), MarketScan's larger cohort of unique patients (approximately 3 times more than PearlDiver) and significantly lower rates of loss to follow-up make it a particularly rich source of data. Truven Health Analytics MarketScan data sets are publicly available to researchers for a fee per year of data. The MarketScan database contains International Classification of Diseases, Ninth Revision, Clinical Modification and Tenth Revision, Clinical Modification, Current Procedural Terminology (CPT), Diagnosis Related Group codes, as well as National Drug Codes. Stanford University Department of Public Health Sciences provided the data source for this study.

\section{Inclusion Criteria}

This study identified patients who underwent primary arthroscopic femoral osteoplasty (CPT 29914) or acetabuloplasty (CPT 29915) between 2007 and 2016. Patients who underwent both were categorized as "both" and included in a subgroup analysis. Only patients with confirmed laterality were included in this study to assure any dislocation or fracture occurred on the surgical side. Individual demographic information and comorbidity status including age, sex, history of diabetes, hyperlipidemia, hypertension, obesity, osteoporosis, and tobacco use of each patient were gathered (Table 1).

\section{Outcomes}

The primary outcome of this study was to determine postoperative complication profiles of patients undergoing femoral osteoplasty (femoroplasty or cheilectomy) or acetabuloplasty. The presence of a postoperative complication was defined as a complication occurring within 30 days of the index surgery (Table 2). These included bursitis (727.3), infection (998.5-998.59, 730.0-730.91, 996.66, 996.67), hematoma $(998.1,998.11,998.12,998.13)$, nerve injury (955.0-955.9, 907.4), or wound complications (998.3, 998.31, 998.32, 998.81, 998.83, 998.4, 101.40, 101.60, 101.80, deep vein thrombosis (451.0-453.9), and pulmonary embolism (415.1-415.19). Thromboembolism was defined as presence of either a pulmonary embolism or deep vein thrombosis. Hip dislocation (835.0835.13), hip fracture (820.0-820.9), and heterotopic ossification $(728,1,728.19,728.13,726.91)$ were included if they occurred within 90 days of surgery. Hip fractures or dislocations were only counted if they occurred on the ipsilateral hip following surgery. 
Table 1. Demographics of Patients

\begin{tabular}{lccr}
\hline & Acetabuloplasty & Femoroplasty & \\
Variables (\%) & Only $\mathrm{n}=2,564$ & Only $\mathrm{n}=11,245$ & $P$ Value \\
\hline Age, y, mean & 36.7 & 36.2 & .3264 \\
Female sex & 76.8 & 61.2 & $\mathbf{< . 0 0 0 1}$ \\
Diabetes & 7.7 & 6.8 & $\mathbf{< . 0 0 0 1}$ \\
Hypertension & 21.8 & 18.1 & $\mathbf{< . 0 0 0 1}$ \\
Hyperlipidemia & 23.9 & 21.3 & $\mathbf{. 0 0 5 4}$ \\
Obesity & 11.9 & 10.2 & $\mathbf{. 0 0 8 6}$ \\
Osteoporosis & 8.1 & 5.4 & $\mathbf{< . 0 0 0 1}$ \\
Tobacco use & 8.1 & 7.6 & .4628 \\
\hline
\end{tabular}

Bold value indicates statistically significant.

\section{Patient Groups and Statistical Analysis}

Patients were stratified into surgical groups based on their index procedure: isolated femoral osteoplasty or acetabuloplasty. A subgroup analysis of patients who underwent both procedures simultaneously was also conducted. Two-sample $t$ tests and $\chi^{2}$ tests were used to assess significant differences in unadjusted demographic data, and postoperative complications. To minimize the effect of potential confounding on the direct comparison of patients undergoing the femoral osteoplasty or acetabuloplasty procedures, a multivariate logistic regression controlled for baseline covariates such as age, sex, and comorbidities that had statistically significant different differences. (Tables 3 and 4). An alpha value of 0.05 was set as significant.

\section{Results}

\section{Patient Cohort}

A total of 13,809 patients met the inclusion criteria of this study, with each group being mutually exclusive (Table 1). Patients were similarly aged among the procedure groups. Patients who underwent acetabuloplasty only were more likely to be female $(76.8 \%)$ than those undergoing femoral osteoplasty $(61.2 \%)(P<$ $.0001)$. Rates of diabetes, hypertension, and hyperlipidemia were significantly higher in the acetabuloplasty cohort versus the femoroplasty group $(P<.05)$.

\section{Complications}

Unadjusted complications rates were similar between the 2 surgical techniques (acetabuloplasty 17.1\%, femoral osteoplasty $19.9 \%, P=.0622$ ). Heterotopic ossification was significantly more common in the femoroplasty cohort ( $11.3 \%$ vs $8.8 \%, P<0.0001)$. Hip fractures were also more common in patients who underwent femoroplasty $(2.4 \%)$ than acetabuloplasty $(2.0 \%)(P<.05)$. Rates of thromboembolism, nerve injury, infection, and hematoma were similar among surgical techniques (Table 2). In the subgroup analysis, a combined acetabuloplasty-femoroplasty was associated with the greatest unadjusted composite complication rate $(21.6 \%$ vs femoroplasty $19.9 \%$ vs acetabuloplasty $17.1 \%, P<.0001$ ) (Table 5).

\section{Multivariate Regression Analysis}

Age groups were at similar risks for hip dislocation, from younger than 20 years to older than 60 years $(P>.05)$. Sex and baseline comorbidity status (diabetes, hypertension, osteoporosis, tobacco use) did not impact risk for hip dislocation. When compared with acetabuloplasty, femoroplasty did not lead to greater rates of dislocation after we controlled for other variables (odds ratio [OR] 0.8, 95\% confidence interval [CI] 0.4-1.1) (Table 3).

Patients who were aged younger than 20 years old were significantly less likely to fracture their hips postoperatively than patients aged $60+$ years (OR 0.3, 95\% CI 0.1-0.8). Female patients were also at lower odds of hip fracture compared with their male counterparts (OR 0.6, 95\% CI 0.5-0.9). Hypertension was independently associated with increased odds of hip fracture (OR 1.7, 95\% CI 1.2-3.5). Femoroplasty or acetabuloplasty did not independently lead to increased or decreased odds of hip fracture $(P>.05)$ (Table 4$)$.

\section{Discussion}

This study sought to better understand the risk factors for these 2 complications. We found that hypertension increased odds for fracture, whereas female sex and younger age was protective against a fracture. However, no covariates independently increased or decreased risk for postoperative hip dislocation. Femoroplasty and acetabuloplasty are common ways to treat cam and pincer FAI, respectively. ${ }^{4,5}$ Proximal femoral fracture and hip dislocation are 2 uncommon, yet major, complications that can occur following these arthroscopic surgeries. ${ }^{15,16,18-20}$ FAI is characterized by abnormal bony morphology of the femoral head and/or the acetabulum as well as abnormal orientation of these 2 features resulting in abnormal contact within the joint

Table 2. Unadjusted Complications Within 30 Days

\begin{tabular}{lccr}
\hline & \multicolumn{3}{c}{ Femoroplasty } \\
\multicolumn{1}{c}{ Variables (\%) } & $\begin{array}{c}\text { Acetabuloplasty } \\
\text { Only } \mathrm{n}=2,564\end{array}$ & $\mathrm{n}=11,245$ & $P$ Value \\
\hline Any complication & 17.1 & 19.9 & .0622 \\
Bursitis & 4.2 & 3.4 & $\mathbf{. 0 3 6 2}$ \\
Hip dislocation (90 d) & 2.3 & 2.1 & .0501 \\
Hip fracture (90 d) & 2.0 & 2.4 & $\mathbf{. 0 3 0 2}$ \\
Hematoma & 1.3 & 0.8 & .0803 \\
Infection & 1.6 & 1.2 & .0857 \\
Nerve injury & 0.3 & 0.3 & .8928 \\
Heterotopic ossification & 8.8 & 11.3 & $<.0001$ \\
$\quad$ (90 d) & & & \\
Thromboembolism & 1 & 1.1 & .4877 \\
Wound complication & 0.8 & 0.7 & .762 \\
\hline Bold value indicates statistically significant.
\end{tabular}


Table 3. Multivariate Logistic Regression to Identify Predictors of Hip Dislocation

\begin{tabular}{lccc}
\hline \multicolumn{1}{c}{ Variables (\%) } & OR & $95 \%$ CI & $P$ Value \\
\hline Age, y (reference 60 y and up) & & & \\
$\quad 0-20$ & 0.4 & $0.1-1.15$ & .051 \\
$21-30$ & 0.7 & $0.2-1.7$ & .2225 \\
$31-40$ & 1.1 & $0.3-2.9$ & .0961 \\
$41-50$ & 0.8 & $0.2-2.0$ & .8521 \\
$51-60$ & 1.1 & $0.3-3.1$ & .7911 \\
Female vs male & 0.8 & $0.6 .-1.1$ & .2468 \\
Diabetes & 0.9 & $0.5-1.7$ & .8134 \\
Hypertension & 1.1 & $0.7-1.6$ & .6732 \\
Hyperlipidemia & 1 & $0.6-1.6$ & .6783 \\
Obesity & 1.2 & $0.8-1.9$ & .4005 \\
Osteoporosis & 1.6 & $0.9-2.8$ & .0931 \\
Tobacco use & 1.3 & $0.7-2.1$ & .2783 \\
Femoroplasty vs acetabuloplasty & 0.8 & $0.4-1.1$ & .3183 \\
\hline
\end{tabular}

CI, confidence interval; OR, odds ratio.

during motion, particularly during flexion and rotation. $^{21-23}$ This contact can lead to damage of soft tissues within the hip and, over time, potentially osteoarthritis. $^{21,22,24}$ Femoroplasty and acetabuloplasty are used alone or in combination to treat cam-type, pincer-type, or mixed-type FAI, respectively.,5 Mixed-type morphology is believed to be most common, ${ }^{25,26}$ although Cobb et al. ${ }^{27}$ found evidence to contradict this in their series.

Clinical complication rates for arthroscopic treatment of FAI are relatively low. A systematic review by Matsuda et al. ${ }^{28}$ found reported major complication rates up to $5 \%$ and a more recent meta-analysis by Minkara et al. $^{29}$ demonstrated a clinical complication rate of $1.7 \%$. Common minor complications include heterotopic ossification, bursitis, transient nerve injury, and superficial wound complications. ${ }^{8,11,12}$ Notable major complications include proximal femoral fracture, hip dislocation, and avascular necrosis of the femoral head. ${ }^{12}$ Interestingly, when looking at large databases sourced data, Truntzer et al. ${ }^{12}$ and Sochacki et al. ${ }^{11}$ reported significantly greater rates than previously shown from high-volume center studies or systematic reviews. The results demonstrated a notably greater complication rate than these previous studies with an unadjusted 30-day complication rates of $17.1 \%$ or acetabuloplasty and $19.9 \%$ for femoroplasty. Using a large, national dataset has been shown to yield complication rates for hip arthroscopy higher than previously reported in the literature. $^{12}$ This may be due to definitions of complications, or potentially the experience of the surgeon, as most published studies come from high-volume, experienced hip arthroscopists, whereas insurance database studies include hip arthroscopy from nonspecialists in hip arthroscopy. ${ }^{11}$ In addition, this study used a highly inclusive definition of complications, which likely contributed to the increased total complications rate compared with the literature. For example, bursitis was noted as a complication in this study but not included in the meta-analysis by Minkara et al. ${ }^{29}$

Proximal femoral fracture is a serious complication following hip arthroscopy and commonly associated with cam-resection (i.e., femoroplasty). ${ }^{10,11,15,16,30}$ Over-resection of the femoral head, ${ }^{30,31}$ early/excessive postoperative weight-bearing, ${ }^{16,32}$ and trauma ${ }^{32}$ are believed to contribute to fracture following hip arthroscopy. In this study, hypertension showed an equal increase in odds of proximal femoral fracture following surgery. Hypertension has been shown in multiple studies with female cohorts ${ }^{33,34}$ and a metaanalysis $^{35}$ to decrease bone mineral density, but this relationship was not demonstrated universally. ${ }^{34,36}$ Hypertension has additionally been found to increase risk for any fracture ${ }^{37}$ and specifically for hip fracture. $^{34,38}$ Therefore, it is reasonable that hypertension was found in the study to increase odds of proximal femoral fracture following arthroscopy. It also may be a factor that hypertension occurs in greater frequency in older patients, another risk factor hip fracture. This study also found male sex $(\mathrm{OR}=1.7)$ and older age $(60$ years + vs $0-20$ years, $\mathrm{OR}=1.3)$ to be independent predictors of proximal femoral fracture. While female patients are more commonly thought to be at greater risk for hip fracture, this analysis controlled for osteoporosis and used a relatively young cohort. Fractures of all types are more common among men than women aged $<50$ years. ${ }^{39}$ The average age in the cohort was 36.2 years for femoroplasty and 36.5 years for acetabuloplasty, so this epidemiologic trend may contribute. The increase risk for fracture among men could be due to increased likelihood of men to experience a trauma given that younger men more frequently sustain pelvic injuries due to high-energy trauma. ${ }^{40-42}$ In addition, men may be more likely to be active earlier following surgery resulting in fracture from early weight bearing.

Table 4. Multivariate Logistic Regression to Identify Predictors of Hip Fracture

\begin{tabular}{lccc}
\hline \multicolumn{1}{c}{ Variables (\%) } & OR & $95 \%$ CI & P Value \\
\hline Age, y (reference 60 years and up) & & & \\
0-20 & 0.3 & $0.1-0.8$ & $<.0001$ \\
21-30 & 0.7 & $0.2-2.1$ & .6233 \\
31-40 & 0.7 & $0.2-2.1$ & .4481 \\
41-50 & 0.8 & $0.2-2.1$ & .2631 \\
51-60 & 0.7 & $0.2-1.8$ & .9249 \\
Female vs male & 0.6 & $0.5-0.9$ & $\mathbf{. 0 0 3 6}$ \\
Diabetes & 0.8 & $0.4-1.4$ & .4286 \\
Hypertension & 1.7 & $1.2-3.5$ &. $\mathbf{0 0 7 4}$ \\
Hyperlipidemia & 1.1 & $0.7-1.6$ & .7622 \\
Obesity & 1.3 & $0.8-1.9$ & .2201 \\
Osteoporosis & 1.6 & $0.9-2.7$ & .0858 \\
Tobacco use & 1.2. & $0.7-2.0$ & .3322 \\
Femoroplasty vs acetabuloplasty & 1.2 & $0.9-1.4$ & .0981 \\
\hline Bold value indicates statistically significant & &
\end{tabular}

Bold value indicates statistically significant. 
Table 5. Subgroup Analysis Complications in Non-Mutually Exclusive Cohort

\begin{tabular}{|c|c|c|c|c|}
\hline Variables (\%) & Acetabuloplasty $\mathrm{n}=2564$ & Femoroplasty $\mathrm{n}=11,245$ & Both $\mathrm{n}=10,026$ & $P$ Value \\
\hline Any complication & 17.1 & 19.9 & 21.6 & $<.0001$ \\
\hline Bursitis & 4.2 & 3.4 & 3.6 & .1817 \\
\hline Hip dislocation & 2.3 & 2.1 & 1.6 & .0216 \\
\hline Hematoma & 1.3 & 0.8 & 0.8 & .0901 \\
\hline Infection & 1.6 & 1.2 & 1.1 & .0749 \\
\hline Nerve injury & 0.3 & 0.3 & 0.4 & .8811 \\
\hline Wound complication & 0.8 & 0.7 & 0.9 & .3915 \\
\hline
\end{tabular}

Bold value indicates statistically significant.

The increase in odds for proximal femoral fracture with advancing age is in keeping with the study by Nguyen et al. that demonstrated age was an independent risk factor for fracture in nonosteoporotic cohort. ${ }^{43}$ These predictors of proximal femoral fracture following femoroplasty and acetabuloplasty provide arthroscopic hip surgeons a better understanding each patient's unique risk factors to enable more nuanced operative decision making (e.g., less resection of femoral head in an older, hypertensive patient) and postoperative planning (e.g., delaying weight-bearing in a patient who is at high risk for fracture).

Hip dislocation following surgery is considered a rare, but serious complication following FAI surgery. Truntzer et al. ${ }^{12}$ reported lower rate of hip dislocation in a broader hip arthroscopy cohort of $0.58 \%$ with 1 -year follow-up. Reported risk factors for dislocation following hip arthroscopy include ligamentous laxity, ${ }^{41}$ iliopsoas tenotomy, ${ }^{43}$ excessive capsular resection, ${ }^{42-45}$ and excessive acetabular resection. ${ }^{18,20,46,47}$ Overresection may lead to hip dysplasia or potentially compromise of the socket and increase ligament laxity by severing capsular insertion into the acetabulum. Some patients may have increased laxity in their ligaments, further increasing their likelihood of a postoperative hip dislocation. ${ }^{4-51}$ Unfortunately, the data do not provide operative reports, so information regarding extent of resection-how many patients had interportal capsulotomies versus $\mathrm{T}$ capsulotomies and how many had partial or complete capsular closures-is unavailable.

In this analysis, after 30 days, femoroplasty-only patients had greater overall unadjusted complication rates compared to acetabuloplasty-only patients $(19.9 \%$ vs $17.1 \%$, respectively). These results differ from those reported by Hartwell et al., ${ }^{13}$ where no difference in complication rates were found. The study's cohort is larger than the cohort in the analysis by Hartwell et al. ${ }^{13}$ (2,564 vs 103 acetabuloplasty cases and 11,245 vs 527 femoroplasty cases, respectively), which may have allowed the analysis to realize differences in complication rates that the Hartwell et al. analysis did not. Femoroplasty did show statistically significant difference in proximal femoral fracture $(2.4 \%$ vs acetabuloplasty at $2.0 \%, P<.050)$. Based on reports of femoroplasty resulting in fracture, the increased rates of proximal femoral fracture are consistent and even expected. In an effort to avoid serious complications associated with femoroplasty (fracture and avascular necrosis) in FAI arthroscopic treatment, Tjong et al. ${ }^{14}$ demonstrated that combined-type FAI can be successfully treated with only acetabuloplasty, but the results from the study raise questions as to the necessity of attempting to avoid femoroplasty. However, a concern with the acetabuloplasty-only technique is the potential for iatrogenic dysplasia. The loss of bony constraint and reduced acetabular coverage may lead to further capsular laxity, hip instability, including dislocation, and ultimately may results in osteoarthritis. ${ }^{52}$ Surgeons may consider the predictors of proximal femoral fracture described in this study when deciding whether avoiding femoroplasty is the best option for any particular case.

\section{Limitations}

While large databases provide a large cohort for analysis, there are notable limitations to this approach. This database does not include many clinically relevant details, such as the amount of bone resected, capsulotomy and management, surgeon experience, postoperative plan, and acute causes of the complications (e.g., traumatic accident). Operative factors including the fidelity of the surgery, involving factors such as femoral bone resection or acetabular bone resection, particularly in comparison with the severity of the FAI, as well as capsular management do not currently exist in these large, publicly available databases, as well as in previous studies. These factors have been demonstrated to contribute to postoperative proximal femoral fracture and hip dislocation. ${ }^{32,44,46,49}$ Given the relatively rare rates of these major complications, there is a greater potential for these clinically relevant details to impact this analysis. To mitigate this, we used a large cohort which should mute the effects of rare events 
such as traumatic injury. An additional limitation of this study is that the cohorts had statistically significant differences in sex and rates of diabetes, hypertension, hyperlipidemia, and obesity. That said, we conducted a multivariate analysis to control for the cohort differences and identify independent predictors. Lastly, the MarketScan database consists of only privately insured patients, and therefore may differ in significant ways from a publicly insured population.

\section{Conclusions}

Older age, male sex, and hypertension all carry increased risk for a hip fracture following acetabuloplasty or femoroplasty. Patient- and procedure-specific factors that could be assessed with this database did not influence risk for hip dislocation,

\section{References}

1. Bonazza NA, Homcha B, Liu G, Leslie DL, Dhawan A. Surgical trends in arthroscopic hip surgery using a large national database. Arthroscopy 2018;34:1825-1830.

2. Montgomery SR, Ngo SS, Hobson T, et al. Trends and demographics in hip arthroscopy in the United States. Arthroscopy 2013;29:661-665.

3. Sing DC, Feeley BT, Tay B, Vail TP, Zhang AL. Age-related trends in hip arthroscopy: A large cross-sectional analysis. Arthroscopy 2015;31:2307-2313.

4. Jackson TJ, Stake CE, Trenga AP, Morgan J, Domb BG. Arthroscopic technique for treatment of femoroacetabular impingement. Arthrosc Tech 2013;2:e55-e59.

5. Pun S, Kumar D, Lane NE. Femoroacetabular impingement. Arthritis Rheumatol 2015;67:17-27.

6. Tannast M, Siebenrock KA, Anderson SE. Femoroacetabular impingement: Radiographic diagnosis-what the radiologist should know. AJR Am J Roentgenol 2007; 188:1540-1552.

7. Mascarenhas VV, Rego P, Dantas P, et al. Imaging prevalence of femoroacetabular impingement in symptomatic patients, athletes, and asymptomatic individuals: A systematic review. Eur J Radiol 2016;85:73-95.

8. Frank JM, Harris JD, Erickson BJ, et al. Prevalence of femoroacetabular impingement imaging findings in asymptomatic volunteers: A systematic review. Arthroscopy 2015;31:1199-1204.

9. Röling MA, Mathijssen NM, Bloem RM. Incidence of symptomatic femoroacetabular impingement in the general population: A prospective registration study. J Hip Preserv Surg 2016;3:203-207.

10. Weber AE, Harris JD, Nho SJ. Complications in hip arthroscopy. Sports Med Arthrosc 2015;23:187-193.

11. Sochacki KR, Jack RA 2nd, Safran MR, Nho SJ, Harris JD. There is a significant discrepancy between "big data" database and original research publications on hip arthroscopy outcomes: A systematic review. Arthroscopy 2018;34:1998-2004.

12. Truntzer JN, Hoppe DJ, Shapiro LM, Abrams GD, Safran M. Complication rates for hip arthroscopy are underestimated: A population-based study. Arthroscopy 2017;33:1194-1201.
13. Hartwell MJ, Morgan AM, Johnson DJ, et al. Risk factors for 30-day readmission following hip arthroscopy. Knee Surg Sports Traumatol Arthrosc 2020;28:1290-1295.

14. Tjong VK, Gombera MM, Kahlenberg CA, et al. Isolated acetabuloplasty and labral repair for combined-type femoroacetabular impingement: Are we doing too much? Arthroscopy 2017;33:773-779.

15. Zimmerer A, Sobau C, Ries C, Miehlke W. Femoral neck fracture is a rare complication after arthroscopic femoroplasty for femoroacetabular impingement with no known prognostic factors and few reported risk factors: A systematic review. J ISAKOS 2017;2:147-151.

16. Ayeni OR, Bedi A, Lorich DG, Kelly BT. Femoral neck fracture after arthroscopic management of femoroacetabular impingement: A case report. J Bone Joint Surg Am 2011;93:e47.

17. JN, Shapiro LM, Hoppe DJ, Abrams GD, Safran MR. Hip arthroscopy in the United States: An update following coding changes in 2011. J Hip Preserv Surg 2017;4: 250-257.

18. Yeung M, Simunovic N, Belzile E, Philippon MJ, Ayeni OR. Systematic review gross instability after hip arthroscopy: An analysis of case reports evaluating surgical and patient factors. Arthroscopy 2016;32:1196-1204.

19. Wylie JD, Beckmann JT, Aoki SK. Dislocation after hip arthroscopy for cam-type femoroacetabular impingement leading to progressive arthritis: A case report. JBJS Case Connect 2015;5:e80-e87.

20. Duplantier NL, McCulloch PC, Nho SJ, Mather RC, Lewis BD, Harris JD. Hip dislocation or subluxation after hip arthroscopy: A systematic review. Arthroscopy 2016;32: 1428-1434.

21. Ganz R, Parvizi J, Beck M, Leunig M, Nötzli H, Siebenrock KA. Femoroacetabular impingement: A cause for osteoarthritis of the hip. Clin Orthop Relat Res 2003; (417):112-120.

22. Sankar WN, Nevitt M, Parvizi J, Felson DT, Agricola R, Leunig M. Femoroacetabular impingement: Defining the condition and its role in the pathophysiology of osteoarthritis. J Am Acad Orthop Surg 2013;21:S7-S15 (suppl).

23. Agricola R, Weinans H. What is femoroacetabular impingement. Br J Sports Med 2016;50:196-197.

24. Agricola R, Waarsing JH, Arden NK, et al. Cam impingement of the hip-a risk factor for hip osteoarthritis. Nat Rev Rheumatol 2013;9:630-634.

25. Beck M, Kalhor M, Leunig M, Ganz R. Hip morphology influences the pattern of damage to the acetabular cartilage. Femoroacetabular impingement as a cause of early osteoarthritis of the hip. J Bone Joint Surg Br 2005;87: 1012-1018.

26. Allen D, Beaulé PE, Ramadan O, Doucette S. Prevalence of associated deformities and hip pain in patients with cam-type femoroacetabular impingement. J Bone Joint Surg Br 2009;91:589-594.

27. Cobb J, Logishetty K, Davda K, Iranpour F. Cams and pincer impingement are distinct, not mixed: The acetabular pathomorphology of femoroacetabular impingement. Clin Orthop Relat Res 2010;468:2143-2151.

28. Matsuda DK, Carlisle JC, Arthurs SC, Wierks CH, Philippon MJ. Comparative systematic review of the open dislocation, mini-open, and arthroscopic surgeries for 
femoroacetabular impingement. Arthrosopy 2011;27: 252-269.

29. Minkara AA, Westermann RW, Rosneck J, Lynch TS. Systematic review and meta-analysis of outcomes after hip arthroscopy in femoroacetabular impingement. Am J Sports Med 2019;47:488-500.

30. Papavasiliou AV, Bardakos NV. Complications of arthroscopic surgery of the hip. Bone Joint Res 2012;1:131-144.

31. Merz MK, Christoforetti JJ, Domb BG. Femoral neck fracture after arthroscopic femoroplasty of the hip. Orthopedics 2015;38:e696-e700.

32. Mardones RM, Gonzalez C, Chen Q, Zobitz M, Kaufman KR, Trousdale RT. Surgical treatment of femoroacetabular impingement: Evaluation of the effect of the size of the resection. J Bone Joint Surg Am 2005;87:273-279.

33. Horner NS, Vikas K, MacDonald AE, Naendrup JH, Simunovic N, Ayeni OR. Femoral neck fractures as a complication of hip arthroscopy: A systematic review. J Hip Preserv Surg 2017;4:9-17.

34. Cappuccio FP, Meilahn E, Zmuda JM, Cauley JA. High blood pressure and bone-mineral loss in elderly white women: a prospective study. Study of Osteoporotic Fractures Research Group. Lancet 1999;354:971-975.

35. Yang S, Nguyen ND, Center JR, Eisman JA, Nguyen TV, Association between hypertension and fragility fracture: A longitudinal study. Osteoporos Int 2014;25:97-103.

36. Ye Z, Lu H, Liu P. Association between essential hypertension and bone mineral density: A systematic review and meta-analysis. Oncotarget 2017;8:68916-68927.

37. Javed F, Khan SA, Ayers EW, et al. Association of hypertension and bone mineral density in an elderly African American female population. J Natl Med Assoc 2012;104:172-178.

38. Vestergaard P, Rejnmark L, Mosekilde L. Hypertension is a risk factor for fractures. Calcif Tissue Int 2009;84:103-111.

39. Sennerby U, Melhus H, Gedeborg R, et al. Cardiovascular diseases and risk of hip fracture. JAMA 2009;302: 1666-1673.

40. Buford TW. Hypertension and aging. Ageing Res Rev 2016;26:96-111.

41. Court-Brown CM, Caesar B. Epidemiology of adult fractures: A review. Injury 2006;37:691-697.
42. Ježek M, Džupa V. The influence of patient age and mechanism of injury on the type of pelvic fracture: Epidemiological study. Acta Chir Orthop Traumatol Cech 2012;79:65-68 [in Czech].

43. Nguyen ND, Eisman JA, Center JR, Nguyen TV. Risk factors for fracture in nonosteoporotic men and women. J Clin Endocrinol Metab 2007;92:955-962.

44. Ranawat AS, McClincy M, Sekiya JK. Anterior dislocation of the hip after arthroscopy in a patient with capsular laxity of the hip. J Bone Joint Surg Am 2009;91:192-197.

45. Sansone M, Ahldén M, Jónasson P, Swärd L, Eriksson T, Karlsson J. Total dislocation of the hip joint after arthroscopy and ileopsoas tenotomy. Knee Surg Sport Traumatol Arthrosc 2013;21:420-423.

46. Prietzel T, Hammer N, Schleifenbaum S, et al. The impact of capsular repair on the dislocation rate after primary total hip arthroplasty: A retrospective analysis of 1972 cases. Z Orthop Unfall 2014;152:130-143 [in German].

47. Jurkutat J, Zajonz D, Sommer G, et al. The impact of capsular repair on the risk for dislocation after revision total hip arthroplasty - a retrospective cohort-study of 259 cases. BMC Musculoskelet Disord 2018;19:314.

48. Matsuda DK. Acute iatrogenic dislocation following hip impingement arthroscopic surgery. Arthroscopy 2009;25: 400-404.

49. Edwards PK, Ebert JR, Joss B, et al. Patient characteristics and predictors of return to sport at 12 months after anterior cruciate ligament reconstruction: The importance of patient age and postoperative rehabilitation. Orthop $J$ Sports Med 2018;6:2325967118797575.

50. Austin DC, Horneff JG 3rd, Kelly JD 4th. Anterior hip dislocation 5 months after hip arthroscopy. Arthroscopy 2014;30:1380-1382.

51. Lima LC, do Nascimento RA, de Almeida VM, Façanha Filho FA. Epidemiology of traumatic hip dislocation in patients treated in Ceará, Brazil. Acta Ortop Bras 2014;22: 151-154.

52. Park MS, Yoon SJ, Kim YJ, Chung WC. Hip arthroscopy for femoroacetabular impingement: The changing nature and severity of associated complications over time. Arthroscopy 2014;30:957-963. 